\title{
Barriers to End-User Designers of Augmented Fabrication
}

\author{
Chandan Mahapatra \\ Rochester Institute of Technology \\ Rochester, NY, USA \\ cm6463@rit.edu \\ Michael McQuaid \\ Rochester Institute of Technology \\ Rochester, NY, USA \\ mickmcquaid@gmail.com
}

\author{
Jonas Kjeldmand Jensen \\ University of Copenhagen \\ Copenhagen, Denmark \\ jkj@di.ku.dk \\ Daniel Ashbrook \\ University of Copenhagen \\ Copenhagen, Denmark \\ dan@di.ku.dk
}

\begin{abstract}
Augmented fabrication is the practice of designing and fabricating an artifact to work with existing objects. Although common both in the wild and as an area for research tools, little is known about how novices approach the task of designing under the constraints of interfacing with real-world objects. In this paper, we report the results of a study of fifteen novice end users in an augmented fabrication design task. We discuss obstacles encountered in four contexts: capturing information about physical objects, transferring information to 3D modeling software, digitally modeling a new object, and evaluating whether the new object will work when fabricated. Based on our findings, we suggest how future tools can better support augmented fabrication in each of these contexts.
\end{abstract}

\section{CCS CONCEPTS}

\section{- Human-centered computing $\rightarrow$ Empirical studies in} HCI.

\section{KEYWORDS}

augmented fabrication; 3D printing; 3D design

\footnotetext{
Permission to make digital or hard copies of all or part of this work for personal or classroom use is granted without fee provided that copies are not made or distributed for profit or commercial advantage and that copies bear this notice and the full citation on the first page. Copyrights for components of this work owned by others than the author(s) must be honored. Abstracting with credit is permitted. To copy otherwise, or republish, to post on servers or to redistribute to lists, requires prior specific permission and/or a fee. Request permissions from permissions@acm.org. CHI 2019, May 4-9, 2019, Glasgow, Scotland UK

(c) 2019 Copyright held by the owner/author(s). Publication rights licensed to ACM.

ACM ISBN 978-1-4503-5970-2/19/05 ..\$15.00

https://doi.org/10.1145/3290605.3300613
}

ACM Reference Format:

Chandan Mahapatra, Jonas Kjeldmand Jensen, Michael McQuaid, and Daniel Ashbrook. 2019. Barriers to End-User Designers of Augmented Fabrication. In CHI Conference on Human Factors in Computing Systems Proceedings (CHI 2019), May 4-9, 2019, Glasgow, Scotland Uk. ACM, New York, NY, USA, 15 pages. https://doi.org/10.1145/ 3290605.3300613

\section{INTRODUCTION}

3D printers are swiftly becoming inexpensive enough to be accessible to the everyday consumer. Much as the introduction of personal computers into the home enabled a new category of "end-user programmer" [33], so 3D printing is bringing into being the end-user maker and a concomitant set of new challenges and opportunities. One of the major activities undertaken by early-adopter end-user makers is augmented fabrication [5]-designing and fabricating new objects that work with existing artifacts. Examples include bicycle pedals [63], equipment for small farms [53], and hand grips for accessibility [12], among many others.

While many researchers have created design tools that enable domain-specific augmented fabrication (see Related Work), the evidence in the literature of novice augmented fabrication design activity is largely incidental to studies of other matters $[3,8,11,13,50,60]$. However, Hudson et al.'s investigation of newcomers to $3 \mathrm{D}$ printing [28] sheds important light on the subject, revealing problems with end-user motivation, domain knowledge, and the use of 3D modeling software. While this work revealed a broad spectrum of challenges encountered by newcomers to 3D printing, the participants in the study varied widely in experience, motivation, software used, and task undertaken. This variance, in combination with the wide scope of the study, makes it difficult to understand the challenges particular to augmented fabrication design tasks, as well as to formulate recommendations for the design of future systems. 
Why study novice behavior in particular? It is our belief that for $3 \mathrm{D}$ printing to become adopted for home use, potential users must be able to not only print objects designed by other people, but objects they design as well. As evidenced in the literature $[11,28,45,60]$, creating new objects to work with existing artifacts is seen as a desirable activity. Our research seeks to uncover factors that may prevent novices from engaging in this activity. While many facets of 3D printing (e.g., design, successful printing, post-processing) may prove challenging to users-as demonstrated by both research [42, 60] and social help sites such as Reddit ${ }^{1}$ and Stack Exchange ${ }^{2}$-in this research we focus on the design part of the process as the first hurdle that end users engaging in augmented fabrication must overcome. The manufactured objects we encounter every day are usually result from the work of engineers and industrial designers; one key question is whether novices possessing none of the expertise of these professionals can come close to the same results when designing an object.

To gain better understanding of the challenges faced by novices designing for augmented fabrication, we conducted a study of fifteen users with little to no prior experience in computer-aided design (CAD). Using the online beginnertargeted 3D CAD tool Autodesk Tinkercad [67], our participants attempted to create a design from scratch, with the goal that the 3D-printed final result work with an existing object. Our objective with this study was to answer the following research questions:

- What obstacles do novice end users encounter when designing for augmented fabrication?

- Where in the process do those obstacles occur?

- What are the impacts of obstacles on the design process?

- To what degree are users able to overcome encountered obstacles?

- What is user perception of the encountered issues?

We are particularly interested in the process that novices follow in designing for augmented fabrication. Inspired by Yung et al. [79], we identified four components of the iterative process of designing for augmented fabrication (Figure 1): capturing information about the physical object, such as measurements; transferring that information into the digital realm; using information about the physical objects to model the new object; and evaluating how the model will interact with the physical world.

Our work extends previous results in end-user design, programming, and electronics, suggesting areas in which future design tools can be improved. The contributions of our paper are to present the first investigation of novice end users in an augmented fabrication design task, to extend

\footnotetext{
${ }^{1}$ https://reddit.com/r/3Dprinting/

${ }^{2}$ http://3dprinting.stackexchange.com/
}

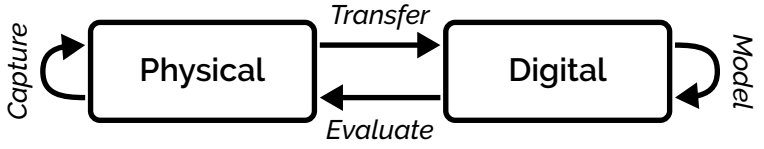

Figure 1: Design flow for augmented fabrication.

the body of knowledge of novice CAD and measurement behavior, and to suggest three areas in which future design tools can be improved for augmented fabrication design.

\section{RELATED WORK}

\section{Augmented Fabrication}

Ashbrook et al. summarized the idea of augmented fabrication as fabrication to work with existing objects [5]. Numerous examples of augmented fabrication can be observed in the wild. The 3D model-sharing site Thingiverse [66] hosts a multitude of downloadable models, many of which are examples of augmented fabrication; Alcock et al.'s study of barriers to using these models [3] incidentally identified multiple augmented fabrication examples, and Chen et al. [18] and Hofmann et al. [27] identify Thingiverse-hosted augmented fabrication examples as part of surveys related to their research. A number of studies around fabrication related to assistive technology have also provided evidence of augmented fabrication design in the wild, including work on volunteers fabricating 3D-printed upper-limb prostheses [52], Buehler et al.'s study of 3D printing in special education classrooms [13] and investigations of augmented-fabrication-based accessibility tools on Thingiverse [11], and McDonald et al.'s demonstrations of how augmented fabrication could benefit patients undergoing physical therapy [43]. There is also evidence that people who are not experts in fabrication may wish to engage in augmented fabrication activity. In their study of potential home users of 3D printers, Shewbridge et al. found that many participants wanted to print objects to repair or improve existing objects [60], and McNally et al.'s cooperative inquiry studies with children revealed similar results [45].

One result particularly related to our work is Kim et al.'s crowdsourced study of problems people have in measuring physical objects [31]-an integral part of the augmented fabrication design process (Figure 1). Their participants measured everyday objects (phone size, laptop screen angle, light bulb diameter) with a variety of tools and variable success, but generally came within $98 \%$ of the correct length measurement. Kim et al. suggested a number of uncertainty accommodation strategies, applied post-design, to allow 3D-printed augmentations to fit even when slightly mis-sized. Our work complements Kim et al. by looking at novice experiences throughout the entire design process, not just in measurement. 
The HCI literature contains a large number of domainspecific augmented fabrication design tools (e.g., [2, 15, 17, 18, 20-22, 25, 36, 37, 57, 64, 69, 79, 80]). These tools typically fall into two broad categories: new techniques for 3D printing, including augmented fabrication; and systems designed to make design for fabrication easier. These systems, however, are generally built with a focus on novel interaction, and are not based on formative studies of users of fabrication technology. Knowledge of how users approach augmented fabrication tasks might lead to further improved design methods, both domain-specific and general purpose.

\section{Studies of End-users}

One way to think about non-professional users of 3D printing is as "end users." Similar to Ko et al.'s definition of end-user programmers as users who "write programs to support some goal in their own domains of expertise" [33], we consider end-user makers as people who use fabrication technology to support their own goals, rather than in a professional capacity. This area has not been well-explored in the literature; however, our methods are informed by previous work on how end users engage with other technologies.

The largest area of research on end-user tool use is around the practices of end-user programmers [9, 14, 16, 41, 51, 58]. Many of the findings in this body of work reveal difficulties specific to the text-based and procedural modality of programming itself; relevant, however, is Ko et al.'s early work [34], which identified six common barriers encountered by students attempting to learn programming. We found that some of the barriers-such as the use barrier ("I don't know how to use [the technology]") [34]-are applicable to enduser designers in augmented fabrication, but that the interaction between physical and virtual entities may introduce further potential confusion, for example in the multi-step process of collecting, transcribing, and entering measurements.

Researchers have also considered end users in other domains. Mellis et al.'s workshop study of non-experts in lowlevel electronics design tasks [46] found barriers similar to Ko et al.'s, including difficulty in debugging circuits. Booth et al.'s study [10] of end users building a circuit using Arduino found what might be called cascading failures, where an early mistake caused a series of hard-to-diagnose issues later in the process. In our study, we observed similar error cascades. Primary among Tetteroo et al.'s issues in end-user development for tangible interaction [65] is the integration of the physical with the virtual. Although taking a different form, we observed similar issues in our study of participants struggling in transitioning between physical and virtual domains.

\section{Novice Behavior}

The behavior of novices in design tasks, and the differences in behavior between novices and experts, has been studied in a variety of fields. Ahmed et al.'s study of novice and expert aerospace-industry designers found that the novice designers used trial and error in the design process, evaluating ideas through implementation and testing rather than analytical reasoning [1]. Several of their novice designers had difficulty with visualizing the final outcome of the design when working in software, and wanted a physical model to aid in understanding. In an augmented fabrication context, the end user will most likely have the physical object they want to design with, but may encounter similar difficulties in visualizing the final outcome.

A common pattern found in design activities undertaken by both experts and novices in design [19], engineering $[6,68]$, and software development [24] is that of "satisficing" [62], or exploring possible solutions only until one that is "good enough" arises. Related to this issue is Ball et al.'s finding that both novice and expert designers "adhere religiously to their unsatisfactory solutions and tended to develop them laboriously by the production of various slightly improved versions until something workable was attained" [6]. We observed this phenomenon amongst our participants to varying degrees.

In contrast to novices, experts more often decompose illdefined problems into smaller, well-defined ones [26]. In the case of augmented fabrication, the problem of designing a new object is ill-defined, but can be broken down into moredefined subtasks such as making measurements, modeling larger parts of an object before proceeding to smaller ones, and leaving out details unnecessary to solving the problem.

\section{D Design \& Printing}

Previous research has investigated non-experts in 3D printing. Hudson et al. observed newcomers to 3D printing in non-makerspace print centers (e.g., in public libraries) to observe the challenges they encountered [28]. They identified problems all throughout the idea-to-print progression; particularly relevant are difficulties users encountered in 3D modeling (often with Tinkercad), including with understanding object locations in space and breaking down modeling into sub-parts. While some of Hudson et al.'s participants engaged in augmented fabrication tasks, the authors do not specifically discuss difficulties in designing for augmented fabrication. Similar general issues are also found in Buehler et al.'s study of 3D printing in special education environments [13]: both student and teacher participants encountered issues with 3D modeling software, including Tinkercad, such as understanding the 3D view, manipulating objects and moving the camera, and positioning objects relative to 
one another. McNally et al. conducted several 3D printingbased cooperative inquiry sessions with children [45]. Their participants identified UI issues such as abstruse buttons and poor tutorials as barriers to using modeling software.

Researchers have also investigated expert users in 3D modeling tasks. Lee et al. surveyed usability issues with professional 3D CAD software to suggest usability principles for complex software of this kind [38]. Grossman et al.'s survey of software learnability [23] used Autodesk AutoCAD, professional-level CAD software, as the basis for the experiment, finding specific issues that generalize into larger categories, including awareness of, locating, and understanding functionality. We observed many examples of similar learnability issues in our participants' interaction with Tinkercad. While we report some of these issues where relevant, the focus of our current research is in the aspects specific to augmented fabrication that cause difficulties in design.

\section{STUDY DESIGN}

In order to understand the problems that novices have in an augmented fabrication design task, we conducted an empirical study in which we requested participants to use Tinkercad to design an object to solve an augmented fabrication task, asking them to design a protective cover for a light switch box of the type common in the United States and Canada (Figure 2). To fully understand the problems that participants encountered and the strategies they used, we recorded audio and video and the computer screen; after completion of each participant's design session, we 3D printed their designed object and invited them to return and comment on their results.

To ensure the task was not insurmountable, one of the authors, experienced in 3D CAD, performed the study as a pilot in three CAD tools of increasing complexity: Tinkercad, SketchUp, and Autodesk Fusion 360. The author was able to accomplish the task in each case in under 15 minutes with no errors. We emphasize, however, that the purpose of this test was simply to ensure that the task could be accomplishedit is not the intent of our research to compare expert and novice performance.

\section{Tinkercad}

Tinkercad is a web-based 3D design environment specifically aimed at children and beginners to 3D modeling. We performed several pilot tests with novices using Tinkercad, SketchUp, and Fusion 360, and found that Tinkercad was by far the easiest software package to work with. Tinkercad is simplified: unlike many professional 3D CAD environments in which the user starts with a series of $2 \mathrm{D}$ "sketches" and measurement constraints, Tinkercad uses only predefined 3D shapes. The fundamental operations (Figure 3) are adding a new shape (A), changing its orientation or location by dragging, scaling via dragging scaling handles or by typing in a measurement (B), and performing boolean operations by grouping solid and hole objects (C, D). Users can add a software ruler which allows adjustment of measurements (E) and relative position $(\mathrm{F})$, and an align tool which can align shapes relative to each other (G). Tinkercad's "workplane," visible as a blue grid in Figure 3, defaults to major lines at $10 \mathrm{~mm}$ and minor at $1 \mathrm{~mm}$; these units and spacing can be changed via a small menu tucked in one corner of the design are of the screen.

\section{Task}

We presented our participants with a motivating scenario: the participant was to imagine they have just moved into a new apartment, and have a three-year-old cousin coming to visit. They have just noticed a missing light switch plate and, worried about the electrical hazard, are going to design and $3 \mathrm{D}$ print something to cover the box. We gave all participants some reference objects, access to Tinkercad's included basic interactive tutorials, a ruler and a digital caliper (both capable of metric and imperial units), a printed tutorial guide for the caliper, and scrap paper and writing materials. We informed participants that they could use any online resources they wished to help them in the task.

We were interested in participant design behavior both when an example of an existing object was available, and when an object had to be designed from scratch. Therefore, we divided our participants into two groups; the only difference between the groups was in the reference objects we provided. We gave participants in the first group access to all of the objects shown in Figure 2: an electrical box with a light switch, a switch plate, and screws. To understand barriers to designing without an object to copy, for the second group we removed the light switch plate from the reference objects, leaving participants with the screws and electrical box with the light switch; we instructed these participants that they could at any time reference existing light switches in the room. Because pilot testing revealed significant difficulty with this task, we elected to place only five participants in the second group, concentrating our understanding on the first.

\section{Participants}

We recruited nine female and six male college students (ages 18-26, mean age 22) via flyers posted on campus. We compensated participants US $\$ 10$ per hour for their time. All participants self-identified as novice users of 3D CAD; three participants reported a minor amount of experience with 3D design tools, but noted that such experience was in the distant past and did not include the software used in the study. Several participants reported experience with 2D graphic 


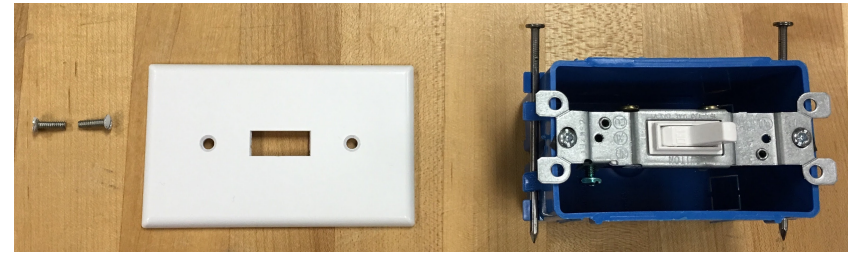

Figure 2: Reference objects for the study: screws, light switch plate, electrical box.

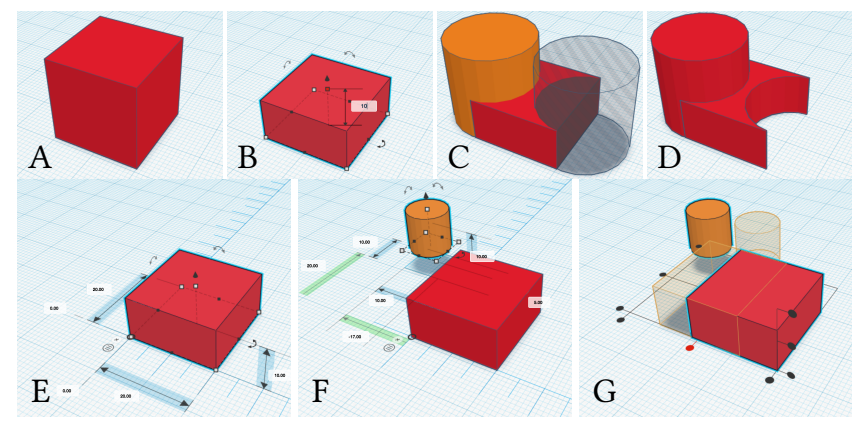

Figure 3: Tinkercad operations. A: a default solid cube; B: the cube, scaled via direct input; C: solid (orange) and hole (semi-transparent/striped) cylinders; D: the cylinders grouped with the scaled cube. E: the Ruler tool placed at the bottom-left corner of the cube, showing dimensions; F: relative placement with the Ruler tool; G: the Align tool showing a preview of alignment.

design software such as Adobe Photoshop or Illustrator, or the UI design tool Sketch. Participants were of various nationalities.

\section{Procedure}

Participants sat at a desk in a makerspace-like lab environment, with several 3D printers and examples of 3D-printed artifacts nearby. Participants used an iMac computer running Tinkercad on the Google Chrome web browser. We used Open Broadcaster Software (OBS) to capture video from the computer screen, the front-facing built-in iMac camera, and an additional USB camera pointing downward at the space in front of and around the computer, with a view of the scrap paper and tools available to the participants.

Before the study, we explained our goals of understanding barriers to augmented fabrication to each participant. We emphasized that we were interested in the process, software, and hardware, and we were not judging their performance. We asked each participant to sign a consent form, and to fill out a brief paper survey detailing their previous experience with design software. We informed participants that they could stop the study when they had decided they were finished with the design or no longer wished to continue.
The study moderator explained the scenario to the participant and started the recording. During the study, the moderator encouraged participants to think aloud, but did not intervene otherwise. The moderator answered questions related to the study structure itself, but not questions directly about how to accomplish the task.

After the participant either determined their design was complete or that they wanted to stop, the moderator conducted a brief post-task interview, asking about difficulties accomplishing the task, features they liked or disliked about the software and hardware, and asking them to reflect on skills required for digital fabrication.

After the interview, we invited each participant whose design was printable (all but one) to return on the following day; we then 3D printed the participant's design. When they returned, we conducted a brief follow-up interview. We asked how they felt about their design, whether they thought they had made mistakes, how close they thought it was to an actual plate, and if they wanted to keep the printed object.

\section{Analysis}

We analyzed the design sessions and the designs themselves in several ways. First, we looked at the degree to which the participant's design could be considered "successful." According to our study scenario, the criteria which participants should have been aiming for was to cover the light switch box to prevent the child from being electrocuted. In the first condition, however, we observed participants explicitly attempting to duplicate the example plate. Therefore, we report several measures of success: 1) whether the design can fit on the light switch box at all; 2) the extent to which the design covers the open areas of the light switch box; and 3) how close the design is to the existing light switch plate. For each of these criteria, we used the digital design in evaluation: although we did 3D-print each design for the post-hoc participant reflection sessions, 3D printers are somewhat variable in their output, so the printed objects may not accurately reflect each designer's intention.

We analyzed the video recordings of the design sessions (approximately 22 hours of video). We first coded the video for participant actions, including watching tutorials; performing in-software shape manipulations such as adding, removing, moving, or scaling objects; using physical measurement tools; manipulating or measuring the reference objects; or using writing tools. Using this coding to assist us, we subsequently looked for patterns in participant actions.

Inspired by Booth et al. [10], we coded the recordings of the design sessions for two broad categories of problems: obstacles and errors. An obstacle is when a participant encounters a problem that needed to be overcome in order to successfully complete the task; an error is an issue in the final design that caused it to fail to function. Often obstacles led 
to errors (e.g., difficulty using the digital calipers could lead to an incorrect size of screw hole). We coded for obstacles in several situations: when the participant evidenced confusion; when the participant struggled with part of the process; and when the participant made an error, we worked backwards in the video to determine actions that led to the error. We coded errors when the final design was unworkable.

Finally, we used thematic analysis [4] to analyze the video recordings of the reflection session, when each participant returned to see their 3D-printed design and discuss their thoughts around the design session.

\section{FINDINGS}

\section{Success}

On average, participants took one hour and 23 minutes to complete the study; this varied widely, however, with a standard deviation of 36 minutes. On average, participants spent about ten minutes using tutorial and online help material; however, the standard deviation is six minutes. P14 spent only 2.5 minutes while P7 spent 20 using this material. There was also wide variation in when during the study participants used the material: while most used the interactive tutorials as the very first step, P1, P4, P7, and P12 each spent several minutes measuring and (with the exception of P12) taking notes before watching the tutorials.

By any of our success criteria, few participants succeeded. Our first criterion, whether the designed object fit on the light switch box at all, largely depends on the three holes being correctly modeled; if they are not, the plate cannot be affixed to the electrical box. As can be seen in Figure 4, many participants struggled with the holes; only those marked with " $\checkmark$ " successfully modeled holes of the correct size and spacing. Common issues with holes included: vertically misaligned; screw hole(s) too small; switch hole too small; wrong spacing between holes; and missing holes.

Aside from hole problems, almost all participants met our second success criterion, creating a plate large enough to cover the electrical box (sized $5.4 \mathrm{~cm} / 21 / 8$ in $\times 10.2 \mathrm{~cm} / 4 \mathrm{in}$ ). The exceptions were designs by P6, P11, and P15; each of these were too small to cover the box.

Our third criterion is how closely the participants matched the model light switch plate. Because participants in the first condition approached the problem by trying to duplicate the existing plate, it is worth considering their success at this attempt. Here we consider only the two-dimensional measurements of the plate, ignoring the thickness, as this value has less impact on the functionality of the design. The image to the right of the design screenshot for each participant in Figure 4 shows a two-dimensional representation of each design overlaid at actual size on a light switch plate and box, and the "Diff" column of Table 2 shows the total

Obstacle
Incorrect use of measurement tool
Making incorrect measurement
Measuring wrong feature or object
Approximation or rounding
Re-measuring the same feature
3D camera causes confusion
3D camera causes manipulation errors

Table 1: Obstacles encountered by participants, according to the area from Figure 1 in which they occurred ("Transition" denotes obstacles with Transferring and/or Evaluating).

difference in measurements between the actual plate and the participant's design. By this measure, several participants had good success-P3, P7, and P8 (successes with respect to the first criterion as well) had minimal deviation.

Surprisingly, we found no statistically significant correlations between difference in measurements and any other factor (using Pearson's method). We tested against study condition, participant gender, participant age, time spent on tutorials, and experience with 2D and 3D software.

On the other hand, when we remove the extreme outlier (P6), the means of "Diff" (Table 2) between the first and second conditions differ significantly as found by a Welch's t-test, $t(6.22)=-3.07, p<0.05$. Using the scheme surveyed by Norman [49, page 185], we classify all the observed errors as knowledge-based mistakes and reflect on Norman's remedy for such mistakes as the furnishing of a good conceptual model. By taking away the physical plate, we deprived the participants in the second condition of a basis for a good conceptual model and the result was significantly more variable measurement. 


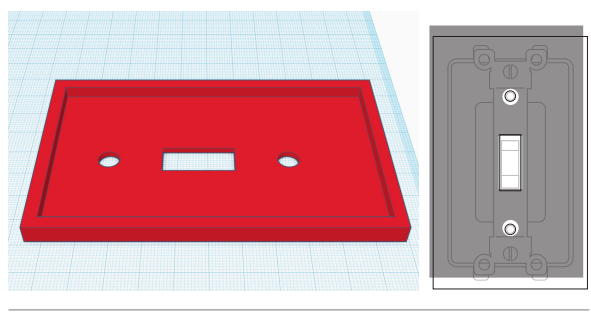

$\mathrm{P} 1 \checkmark(\mathrm{F}, 1: 35)$
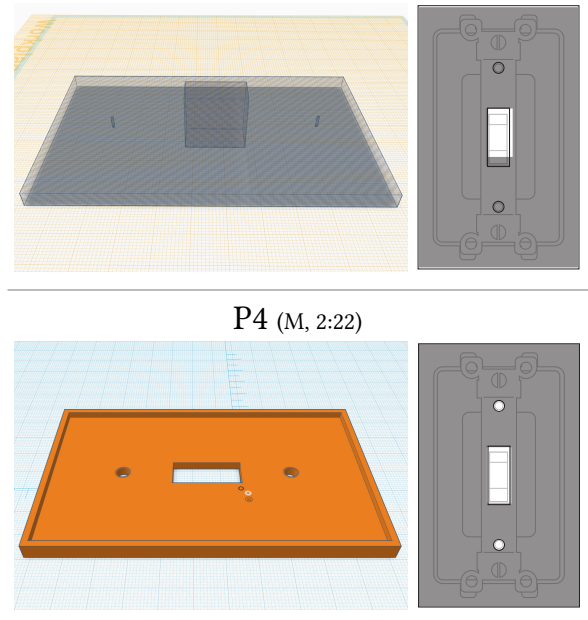

P7 $\checkmark(\mathrm{M}, 1: 27)$

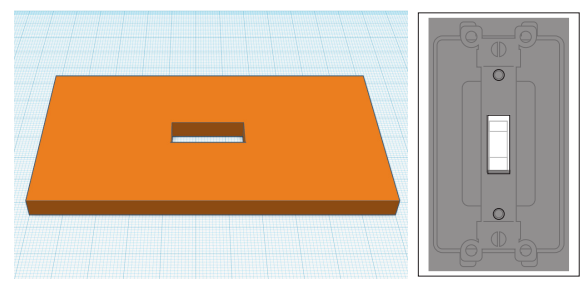

P10 (F, 0:44)

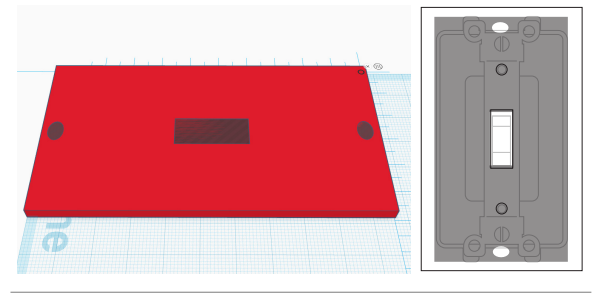

$\mathrm{P} 13(\mathrm{M}, 2: 26)$
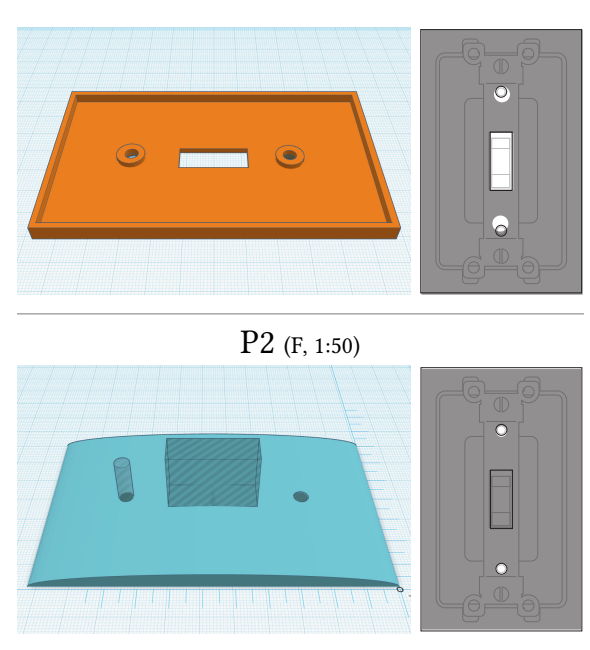

P5 (F, 1:09)

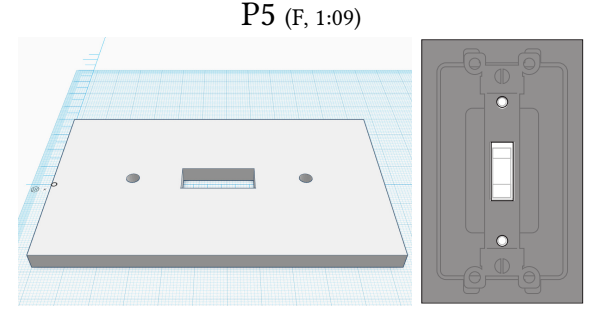

$\mathrm{P} 8 \checkmark(\mathrm{F}, 0: 43)$

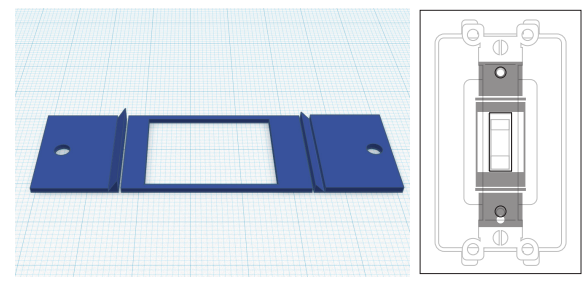

P11 (M, 1:01)

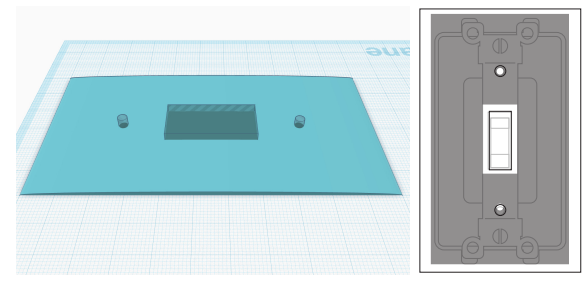

P14 (F, 0:53)

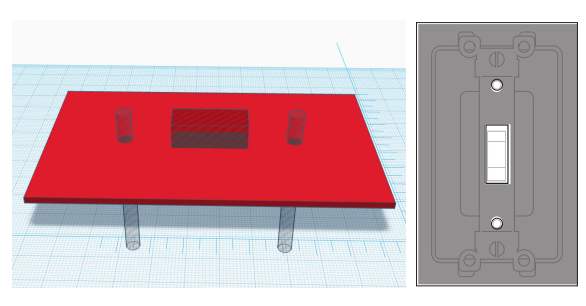

P3 $\checkmark(\mathrm{F}, 1: 41)$
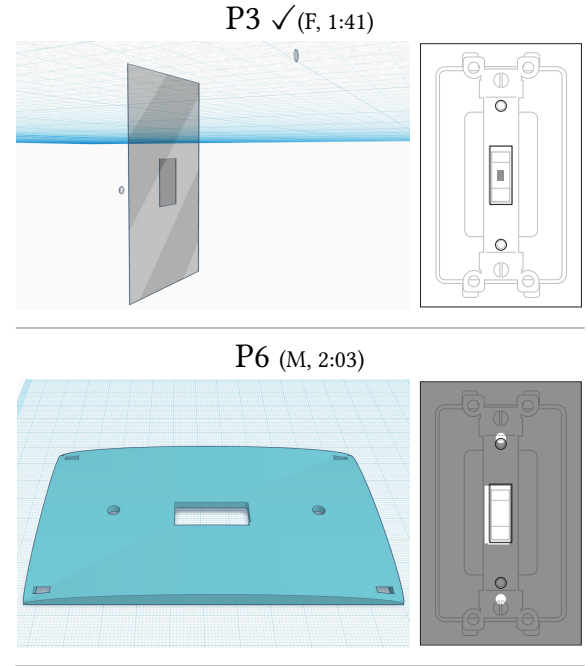

P9 (F, 1:04)

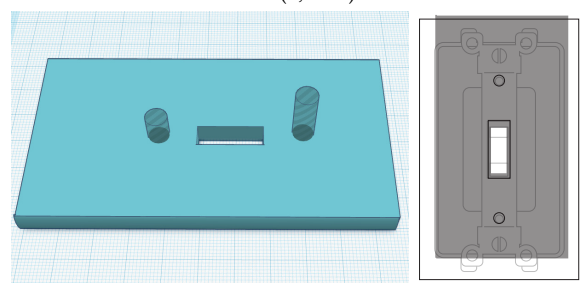

P12 (M, 1:23)

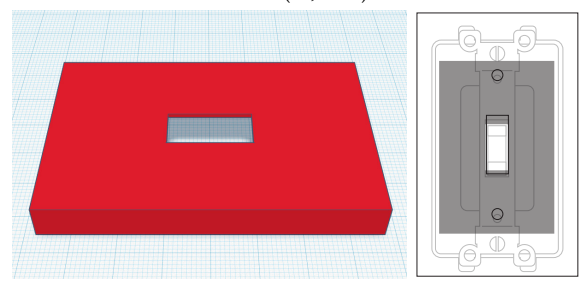

$\mathrm{P} 15(\mathrm{~F}, 0: 22)$

Figure 4: The left image for each participant is a Tinkercad screenshot of the final results from that participant. Colors are as chosen by participants; diagonally-striped semi-transparent areas are possibly ungrouped hole shapes (see Study Design for an explanation of grouping). The perspective for P6 is different to illustrate feature orientations. The right image in each is an overlay of the participant's design (shaded in gray) on top of a $2 \mathrm{D}$ representation of the actual plate (black lines) and switch box (gray lines). Under each pair of images is the participant number, gender, and total study time (HH:MM); “ $\checkmark$ ” indicates a working design. Participants 11-15 did not have the switch plate to reference. 


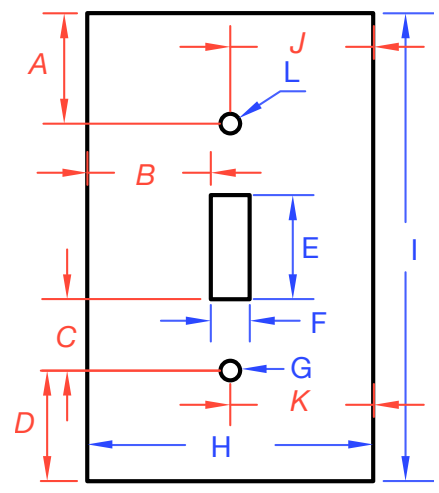

Figure 5: Light switch plate reference dimensions. Letters in red (A$\mathrm{D}, \mathrm{J}, \mathrm{K})$ indicate distance measurements while blue dimensions (E-I, L) indicate size measurements. See Table 2, Ref row, for values.

\begin{tabular}{|c|c|c|c|c|c|c|c|c|c|c|c|c|c|}
\hline & A & B & C & D & E & $\mathrm{F}$ & G & $\mathrm{H}$ & I & $\mathrm{J}$ & $\mathrm{K}$ & $\mathrm{L}$ & Diff \\
\hline Ref & 25.4 & 29.4 & 18.3 & 25.4 & 25.4 & 9.5 & 4.8 & 69.9 & 114.3 & 34.9 & 34.9 & 4.8 & \\
\hline $\mathrm{P} 1$ & 32.3 & 27.6 & 17.6 & 22.4 & 24.1 & 10.8 & 7.4 & 69.6 & 114.1 & 36.7 & 36.7 & 7.4 & 9.1 \\
\hline P2 & 31.5 & 29.9 & 17.9 & 28.9 & 25.0 & 10.0 & 7.0 & 70.0 & 115.0 & 35.7 & 36.0 & 7.0 & 7.9 \\
\hline P3 & 27.0 & 29.0 & 16.5 & 27.0 & 26.0 & 11.0 & 5.0 & 69.0 & 113.0 & 35.0 & 35.0 & 5.0 & 3.7 \\
\hline P4 & 22.3 & 28.4 & 25.7 & 18.3 & 21.0 & 10.6 & 0.6 & 69.0 & 113.0 & 33.3 & 34.7 & 0.6 & 13.3 \\
\hline P5 & 27.2 & 29.3 & 15.4 & 26.5 & 29.8 & 10.5 & 4.9 & 69.2 & 113.6 & 34.6 & 34.6 & 4.9 & 5.8 \\
\hline P6 & 0.6 & 0.5 & 0.2 & 1.4 & 1.0 & 0.7 & 0.2 & 2.8 & 4.5 & 0.4 & 2.3 & 0.2 & 147.9 \\
\hline P7 & 27.1 & 29.7 & 17.7 & 27.1 & 24.5 & 10.7 & 4. & 70.0 & 114.0 & 35.0 & 35.0 & 4.8 & 2.9 \\
\hline P8 & 27.0 & 29.7 & 17.0 & 27.0 & 25.5 & 10.5 & 4.8 & 70.0 & 114.0 & 35.0 & 35.0 & 4.8 & 2.9 \\
\hline P9 & 19.7 & 3 & 20.7 & 24.2 & 25 & 11.0 & 4.5 & 70.0 & 114.0 & 35.1 & 35.1 & 4.5 & 6.7 \\
\hline $\mathrm{P} 10$ & - & 25.5 & - & - & & 10 & - & & 110.0 & - & - & - & 65.0 \\
\hline P11 & 4.5 & 1.0 & 20.6 & 3.3 & 26.1 & 20.0 & 3.4 & 22.0 & 71.8 & 11.0 & 11.0 & 3.4 & 84.2 \\
\hline $\mathrm{P} 12$ & - & 24.0 & - & - & 20.0 & 6.8 & - & 59.0 & 106.9 & - & - & - & 65.9 \\
\hline P13 & 4.3 & 24.0 & 36.7 & 4.7 & 23.2 & 9.9 & 4.8 & 58.0 & 106.3 & 29.0 & 28.6 & 4.7 & 39.1 \\
\hline $\mathrm{P} 14$ & 25.5 & 22.5 & 14.5 & 25.5 & 30.0 & 15.0 & 3.0 & 60.0 & 110.0 & 30.1 & 30.1 & 3.0 & 16.8 \\
\hline P15 & - & 20.0 & - & - & 20.0 & 10.0 & - & 50.0 & 75.0 & - & - & - & 78.5 \\
\hline
\end{tabular}

Table 2: Actual reference (italicized) and per-participant measurements for each dimension in Figure 5, in millimeters. A dash '-' in a cell indicates no measurement for that dimension; for example, missing holes. The bold Diff column is the Euclidean distance between the participant's measurements and the reference, using 0 for missing values.

\section{Errors and Obstacles}

What factors caused participants to succeed or not, according to any of our three criteria? The range of possible errors in the switch plate design is limited: the plate itself could be too small (e.g., P15), or one or more holes could be too small (e.g., P4), missing (e.g., P5's switch hole or P10's screw holes), or misaligned (e.g., P12). Other features of the resulting object could be "incorrect" but not cause the design to fail, such as P14's switch hole being too large.

Of more interest are the obstacles that participants encountered, especially those that led to unworkable designs. We defined obstacles as issues participants encountered while completing the task, and cataloged the most common (summarized in Table 1). Obstacles often, but not always, led to errors that caused unsuccessful designs. The obstacles fall into three general domains: the physical world, the digital modeling environment, and the transition between the two (Figure 1).

Physical Obstacles. Almost all of the obstacles participants encountered in the physical domain related to capturing information about the physical object, or measuring: using measurement tools correctly, making measurements correctly, and measuring the correct part of the correct object. The five participants in the second condition had the additional obstacle of not having the light switch plate, and having to conceive for themselves what to measure to fulfill the task conditions.

Participants had problems with both the caliper and the ruler. Although we provided a booklet of caliper usage techniques, participants still had problems using the tool correctly, often struggling to determine which of the caliper's set of measurement jaws to use for a task. Some participants felt more comfortable with the ruler but still encountered difficulties; for example, $\mathrm{P} 4$ appeared to correctly measure the length of the switch hole (E), but wrote down an incorrect value, and P10's measurement for E is off due to the ruler bending as she measured. Possibly stemming from measurement difficulties, we observed participants very frequently re-measuring the same part of an object, even when they had a dimensioned sketch on their paper.

Participants particularly struggled with measurements involving placement of the screw holes (A, C, D). Participants using the ruler sometimes misread or rounded the measurement, while those using the caliper frequently struggled with how to position the tool jaws correctly to acquire the distance. Often participants became confused about whether their measurement included some of the diameter of the screw hole-although often they were not aware of this confusion.

Another difficulty stemmed from measuring the correct part of the correct object. P1, for example, used several measurements from the light switch box as plate dimensions, even though the existing plate was available to her. P11-P14 only had the box and screws, and used several measurements from these objects directly in their plate design; for example, P11 used the diameter of the screw as the size of the hole, resulting in a slightly-too-small opening, while P13 removed the screws holding the switch to the box (see Figure 2) and replicated the elliptical holes in the switch body.

Digital Obstacles. Many obstacles occurred solely in the digital domain, while modeling the new object. These issues can be broadly viewed as usability problems with Tinkercad, or as the result of participant unfamiliarity with 3D CAD 
software. The obstacles most often encountered, and most likely to cause errors in the final design, fall into three categories: difficulties stemming from the 3D camera view, issues positioning and scaling objects, and problems with finding and understanding software functionality.

One major stumbling block for participants-also seen in previous research $[13,28]$-was around understanding and manipulating the $3 \mathrm{D}$ view of their object. Participants frequently struggled to adjust the view to see the part of their design they were working on, often rotating and zooming over and over again. While these operations were covered in the tutorial, panning the view was not, and no participant discovered how to do so. Some participants avoided manipulating the view as much as possible by operating with the view solely from the top: "Oh! If I look from the top, I should be able to know... how in relationship to... [pointing] this from this and this to this" (P5). While this approach helped P5 to position her shapes, it also led to the error that prevented her design from working: in the top-down view, it became difficult to distinguish the callouts for numerical input from one another. Confused, she accidentally set the Z-position instead of the height of her switch hole object to the thickness of the plate, causing it to rest exactly on top.

Viewpoint changes could also cause direct manipulation errors. Figure 6 shows how manipulation handles (white: size, black: position) "stick" to the object as the viewpoint moves. As an object becomes smaller on the screen, the handles remain the same size, making it easy to accidentally move an object on the $Z$-axis instead of dragging it in the $X Y$-plane. This obstacle was a primary challenge for P6, who spent much of the latter hour of the study manipulating the camera angle and repositioning objects. P2

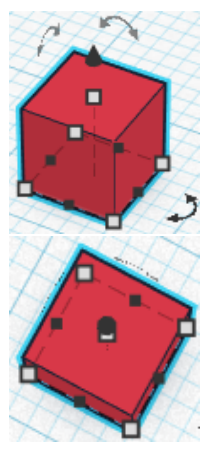

Figure 6 also encountered this difficulty, commenting, "I can't get a good grip [on] the cylinder" as she attempted to move a part and instead accidentally rotated it.

Finally, as noted in previous research [23, 38], awareness of and ability to find functionality can be an issue in CAD software. Our participants encountered these issues as well. For example, during the study, P2 commented: "I wish there was a way... [to measure] from an edge to where this object [is]... I assume there is a way to do that, but I have not figured it out." This issue led to the screw hole mispositioning visible in P2's final design-she in fact had measured correctly to within a half millimeter of measurement C. Frustrated, P4 turned to YouTube for solutions to his problems with precise positioning, searching for "how to move tiny objects in tinkercad", but eventually ending up on one of the Tinkercad tutorials he had already completed.
Physical/Digital Transition Obstacles. Although both physical and digital obstacles contributed to participant issues, the most severe problems resulted from the necessity to work in both of these domains simultaneously, both transferring information about the physical object into Tinkercad, and evaluating whether the design would perform as desired (Figure 1). We observed problems around task decomposition, focus, and understanding the correspondence between the physical and digital.

Simon notes that experts perform a break-down of "illstructured problems" into smaller, more-structured parts [61], while Ho shows that novices to design tasks do not have the knowledge necessary to do so [26]. We also observed this phenomenon: participants had no clear idea of which physical features to model first, nor which were most important to solving the problem in the scenario. We saw participants painstakingly measuring and modeling features from the existing plate such as edge bevels (e.g., P9) or screw hole countersinks (e.g., P2) before having successfully constructed a simple box of the right size. Likely because the task was even more ill-structured, participants in the second condition, without the example light switch plate, suffered even more from this difficulty. P11's design illustrates this problem: he designed several small parts that closely fit the contours of the switch itself, but failed to cover the box itself: "the idea was to have the whole thing as one continuous piece, not five separate components."

Participants were often hyper-focused on one aspect of the task and missed that they made a mistake. A common issue occurred when participants simply eyeballed the placement of objects, forgetting to measure. Often they were able to center the holes relative to the long dimension of the plate, but multiple participant designs failed because of imprecise placement of the three holes relative to each other. For example, after the study, P9 commented, "I realized afterwards, I centered these, these circles [screw holes], but I never measured how far they were supposed to be from the center." Other participants also eyeballed the size of some objects; for example, P4's screw holes-aside from sitting on top of the plate object-are tiny, a result of him simply typing in some apparently random values. P15 is an extreme example of eyeballing: she made no measurements whatsoever, simply guessing at sizes.

One of the most significant obstacles was understanding the correspondence between the two realms: transferring information from the physical to the digital, and evaluating whether the design is correct. This issue often manifested in confusion around measurement units. Although Tinkercad's default unit system is millimeters, participants had difficulty finding this information: when Tinkercad displays measurements (e.g., Figure 3B) it does so without units. P14 
commented, "I assumed that the [workplane] grid was in centimeters instead of millimeters. So, I made the whole object based on millimeters ... so now I have to redo it, using centimeters." P6 illustrates an extreme example: he assumed that because the caliper measured in inches, Tinkercad used the same units, leading to a very tiny model. Treating P6's measurements as inches (i.e., multiplying by 24.5 ), the difference between his design's dimensions and the reference becomes $43.7 \mathrm{~mm}$, well within the range of other designs.

Participants also struggled to place objects relative to one another within Tinkercad. Compared to professional CAD packages, Tinkercad's support for precise positioning is limited. Often participants took a measurement in a way that made sense physically-for example, measuring from the bottom edge of the plate to the closest inside edge of the lower screw hole is simple with the caliper-only to discover that Tinkercad had no facility for positioning objects in the same way. This obstacle led to the necessity to either remeasure a different way or to perform manual calculations; both approaches could lead to error.

\section{Reflection}

We conducted two brief, semi-structured interview sessions with participants. The first was immediately following the design session, when we asked questions about their design process and immediate feelings. We asked them to return the following day, when we presented them with their design 3D-printed, and asked about their satisfaction with their object and to reflect on their process and potential mistakes they might have made. We also gave them the option to keep the 3D-printed object. While the interview content ranged widely, here we report comments that provide understanding additional to our observations of the design sessions themselves.

Design Process. Participant reflection on the design process itself frequently centered around the participant's mental model of how to proceed with the task. P15 said that "it was hard to figure out where to start", and P9 commented on trying to figure how to construct her object: "For example, the top that I made is not flat like this [existing plate], and I can't quite visualize what shapes I would need to use to create that object."

However, most participants understood the basic sets of measurements they needed to make. P11 said, "First I needed to understand what exactly I was going to be designing. So then, looking at the object and using the calipers,...I took individual measurements and then I marked them down on the sheet so that I could refer to them easily." P3 followed a similar procedure: "First I measured the length and width of the outer plate, and then I measured the width of the switch hole, then I tried to make shapes with those measurements in the software program."

Problems and Solutions. A common theme that emerged in the interviews was participant reflection on the problems they encountered. Frequently, measurement was a topic of frustration, both in the physical and the digital realms. A frequent struggle was with trying to position the screw holes in Tinkercad, for both participants without working designs: "I couldn't figure out how do you measure exactly where to put these holes properly" (P4), and those with: "I had to align [the screw holes], and then know the distance between the center and [the switch hole], that was the biggest time consuming process of everything" (P7). P2 suspected undiscovered functionality that would have eased this process; mirroring her comments during the study, she said, "I wish there was a way-there probably is a way!... if I could input a distance from one edge to the side of the [screw] hole!"

Other participants were less sure of what caused the problems in their designs. P5 was puzzled about the missing switch hole in her design: "I don't understand? Because the square [hole] was there. Maybe it didn't have enough depth to it? Or it wasn't linked to the main piece? I dunno. What was it?" P2 was similarly unclear on the misalignment of her screw holes: "Maybe I just mis-drew. Or mis-measured. It's, I think, a millimeter off. Or two. Yeah, it's like two millimeters off?"

Sentiment. Despite the difficulty of the task and the fact that their designs frequently failed to work, participants were very positive about the entire experience, and especially their printed object. P13 said, "I like how it turned out ... it seems like a cool thing to have. The first thing I ever designed in 3D." P15 said, "just to see my work actually, physically, come together, because it's on the screen ... but you don't really actually see it till it's printed, so, I think that's really cool." Even P6, who ended without a printable design, said, "If you are able print out these things, who wouldn't do it? It's really fun to do it ... you can just make it yourself."

\section{DISCUSSION}

In a sense, our results are not surprising: we asked novices to use unfamiliar tools to perform a difficult task, and they struggled to do so. Professional engineers and industrial designers undertake years of training and practice in order to create manufactured objects, while our participants spent on average ten minutes looking at tutorials. From another standpoint, then, the amount of success they achieved is remarkable: almost every participant managed to create an object approximating a light switch plate, even if it was not functional as such. Based on participant reflection in the posthoc interviews, we posit that, given the ability to iterate by 3D-printing, most would achieve success with one or two 
more rounds of design. To improve user experience, however, it would be desirable to reduce the amount of trial-and-error required. Our participants encountered a wide variety of obstacles, occurring in each of the areas illustrated in Figure 1: physical, digital, and in the transitions between. Using these obstacles and each of the transitions indicated by arrows in Figure 1, we suggest four areas of design opportunity to improve user experience with future augmented fabrication systems.

\section{Physical Capture}

The first potential obstacle in an augmented fabrication design task is forming a mental picture of the task itself. What physical properties of the existing object must be capturedor at least be taken into account-to create the new object? In our study, the task itself was well-defined, but participants struggled to break it down into a set of procedural capture steps. Once they started taking measurements, they had difficulty in properly using the tools to measure the objects. Similarly to previous researchers investigating end-user behavior in domains such as software development [34], electronic circuits [46], and physical computing [10], we see a need for more-structured help around augmented fabrication design.

Researchers have begun to investigate methods for helping end users in domains other than programming to learn new tasks; for example, Tinkercad's tutorial system may have been inspired by Li et al.'s gamified tutorial system for AutoCAD [39]. In the physical domain, Knibbe et al.'s Smart Makerspace [32] walks novices through a DIY task and automatically provides context-sensitive instruction and help. This previous research suggests that providing help structured around specific augmented fabrication tasks, and common tools such as digital calipers, might be a powerful aid. One method might take advantage of the property that many objects users may want to augment are manufactured, with common patterns of geometrical relationships [40]. We envision a task-based help system in which a user might select from a library of common shapes and relationships (e.g., a flat shape with a hole in it or an object with curved edges) and receive help on both measuring and modeling such an object.

\section{Physical-to-Digital Transfer}

Beyond providing help around how to properly use tools, a number of researchers have built systems to help users capture information about physical objects and transfer measurements directly to 3D modeling systems. Computer-vision-based systems such as Bend-a-Rule [73] or Nickel for Scale [44] can help capture measurements from photographs, and more-complex systems such as Situated Modeling [36] and MixFab [76] allow the user to include real-world objects as reference points in augmented-reality modeling environments. The drawback of camera-based tools like these is lack of precision in their ability to capture objects.

Another approach is to augment the high-precision measurement tool itself: the button on Weichel et al.'s connected digital caliper [74] copies the current dimension to a CAD program, sidestepping problems with users misreading or mis-transcribing the measurement. A combination of these methods may help end users the most-spatially tracking both the precision tool and the object would allow measurements to be placed next to each other in 3D space, building up a framework of measurement constraints to assist the user in modeling.

\section{Digital Modeling}

While many of the modeling-related obstacles encountered in our study were consequences of usability issues in Tinkercad, many other problems arose due to the nature of the augmented fabrication task. A 3D model created without the need to interconnect with an existing object can have unconnected components, features that are not precisely aligned, and other inconsistencies; objects for augmented fabrication, however, must be more "correct" in order to interface with existing objects. Professional 3D CAD tools such as Fusion 360 provide a wide suite of tools to ensure consistent designs: symmetry can be ensured by mirroring, precise measurements via explicit dimensioning, and alignment via constraints.

While one possibility is to import these tools into novice software, the effect would be a lessening of its "novice-ness," raising the threshold for usability [48]. Another possibility is to constrain the design by incorporating domain-specific knowledge, a common approach in task-specific tools (e.g., $[18,25,59,79])$. A third option may be a novice-centric design environment that is augmented-fabrication-aware. Two-dimensional design tools such as Adobe Illlustrator or Omnigraffle automatically snap drawing components to maintain spatial relationships such as spacing; this kind of feedback could also be of use when modeling to augment a manufactured object. Going further, much like fabrication-aware tools that ensure a model is structurally sound [78] or efficiently printable $[70,72]$, augmented-fabrication-aware tools might check to see if features are almost symmetric, closeto-but-not-quite touching, or just out of alignment.

\section{Digital-to-Physical Evaluation}

A key challenge in augmented fabrication is in understanding whether the model being designed will function as intended when it is fabricated. Our participants struggled with the fit aspect, with many models being unable to be affixed to the light switch box. While many fabrication-aware systems can analyze models for particular functionality such as being 
able to support weight [77], balance [56], or float [71], being able to automatically determine qualities like "fit" requires knowledge of the existing object [27, 29, 35]. One approach in the literature is using a "proxy" material that allows users to model with a tangible substance that fits the shape of the existing object; for example, Jones et al.'s system 3D scans annoted clay models to produce a new object that precisely meshes with the existing one [30], while Weichel et al.'s ReForm [75] both scans and prints clay, enabling the user to quickly modify the soft printed object to better conform to the existing artifact. A promising recent development is "low-fidelity" fabrication, in which a less-detailed representation of the model can be much more quickly printed [47], potentially allowing the user to evaluate the printed model for fit. A refinement of this capability is what Baudisch and Mueller term continuous interactive fabrication [7], where a system prints an object simultaneous with the user's design activity [55].

These systems point towards the potential for fully in-situ augmented fabrication design environments, which "allow the user to work directly with the existing object as part of the design process" [79]. Peng et al.'s RoMA system [54] provides fully in-situ modeling and printing, with an AR view of the model which a printer-wielding robot arm prints in-place as the user designs. A less-complex system, Yung et al.'s Printy3D [79] allows children to design enclosures for simple electronics using projected augmented reality. Both of these systems assist the user in evaluating their design with respect to the real world; however, RoMA requires a large, complex hardware setup, and Printy3D is limited to simple situations where the system already knows about the objects being augmented. There is opportunity for further work in this space, to enable the user to perform all facets of the augmented fabrication design process, integrating capture, transfer, modeling, and evaluation into a single seamless process.

\section{LIMITATIONS}

There are a number of limitations to our study of novice augmented fabrication design activity. Although our participants were diverse in gender and nationality, they were all students at a US university and as such are not representative of the population at large. The design task in the study, chosen for its simplicity and potential to be accomplished in a reasonable amount of time, was not in itself intrinsically motivating to participants; although many enjoyed the design experience, we might observe differing results with augmented fabrication tasks chosen by participants based on their own needs. Finally, we chose design software that is aimed at novices; training novices on more complex software that has better support for augmented-fabrication-related tasks (e.g., symmetry or measurement constraints) might yield further observations of difficulties in 3D modeling.

\section{CONCLUSIONS}

We presented a study of novice engagement with augmented fabrication, the practice of designing and fabricating an artifact to work with an existing object. We investigated novice end-user experiences of designing for augmented fabrication via an experiment in which participants were asked to design a replacement light switch plate. We found obstacles to successful completion in the physical and digital components of the task, and in the transition between the two. To help to overcome the obstacles we identified, we suggest potential improvements in four areas of the augmented fabrication design process: capturing information about physical objects, transferring information to 3D modeling software, digitally modeling a new object, and evaluating whether the new object will work when fabricated.

\section{ACKNOWLEDGEMENTS}

This material is based upon work supported by the National Science Foundation under Grant No. IIS-1464377.

\section{REFERENCES}

[1] Saeema Ahmed, Ken M Wallace, and Luciënne T Blessing. 2003. Understanding the differences between how novice and experienced designers approach design tasks. Research in Engineering Design 14, 1 (Feb. 2003), 1-11.

[2] Yoh Akiyama and Homei Miyashita. 2016. Fitter: A System for Easily Printing Objects that Fit Real Objects. In UIST '16: Adjunct Proceedings of the 29th annual ACM symposium on User interface software and technology. ACM, New York, New York, USA, 129-131.

[3] Celena Alcock, Nathaniel Hudson, and Parmit K Chilana. 2016. Barriers to Using, Customizing, and Printing 3D Designs on Thingiverse. In ACM Conference on Supporting Group Work (GROUP). 1-5.

[4] Jodi Aronson. 1995. A Pragmatic View of Thematic Analysis. The Qualitative Report 2, 1 (1995).

[5] Daniel Ashbrook, Shitao Stan Guo, and Alan Lambie. 2016. Towards Augmented Fabrication: Combining Fabricated and Existing Objects. In Proceedings of the 2016 CHI Conference Extended Abstracts on Human Factors in Computing Systems. ACM, New York, New York, USA, 15101518.

[6] L J Ball, JSBT Evans, I Dennis, and 1994. 1994. Cognitive processes in engineering design: A longitudinal study. Ergonomics 37, 11 (1994), 1753-1786.

[7] Patrick Baudisch and Stefanie Mueller. 2017. Personal Fabrication. Foundations and Trends in Human-Computer Interaction 10, 3-4 (2017), 165-293.

[8] P Blikstein. 2013. Digital fabrication and 'making' in education: The democratization of invention. FabLabs: Of machines (2013).

[9] Tracey Booth and Simone Stumpf. 2013. End-User Experiences of Visual and Textual Programming Environments for Arduino. In EndUser Development. Springer, Berlin, Heidelberg, Berlin, Heidelberg, 25-39.

[10] Tracey Booth, Simone Stumpf, Jon Bird, and Sara Jones. 2016. Crossed Wires: Investigating the Problems of End-User Developers in a Physical Computing Task. In CHI '16: Proceedings of the 2016 CHI Conference 
on Human Factors in Computing Systems. ACM Press, New York, New York, USA, 3485-3497.

[11] Erin Buehler, Stacy Branham, Abdullah Ali, Jeremy J Chang, Megan Kelly Hofmann, Amy Hurst, and Shaun K. Kane. 2015. Sharing is Caring: Assistive Technology Designs on Thingiverse. In CHI '15: Proceedings of the 33rd Annual ACM Conference on Human Factors in Computing Systems. ACM Press, New York, New York, USA, 525-534.

[12] Erin Buehler, Amy Hurst, and Megan Hofmann. 2014. Coming to grips: 3D printing for accessibility. In ASSETS '14: Proceedings of the 16th International ACM SIGACCESS Conference on Computers and Accessibility. ACM, New York, New York, USA, 291-292.

[13] Erin Buehler, Shaun K. Kane, and Amy Hurst. 2014. ABC and 3D: opportunities and obstacles to 3D printing in special education environments. In ASSETS '14: the 16th international ACM SIGACCESS conference. ACM, New York, New York, USA, 107-114.

[14] Jill Cao, Yann Riche, Susan Wiedenbeck, Margaret Burnett, and Valentina Grigoreanu. 2010. End-user mashup programming: through the design lens. ACM, New York, New York, USA.

[15] Patrick Carrington, Shannon Hosmer, Tom Yeh, Amy Hurst, and Shaun K. Kane. 2015. “Like This, But Better”: Supporting Novices' Design and Fabrication of 3D Models Using Existing Objects. In iConference.

[16] Irina Ceaparu, Jonathan Lazar, Katie Bessiere, John Robinson, and Ben Shneiderman. 2004. Determining Causes and Severity of End-User Frustration. International fournal of Human-Computer Interaction 17, 3 (Sept. 2004), 333-356.

[17] Xiang 'Anthony' Chen, Stelian Coros, Jennifer Mankoff, and Scott E Hudson. 2015. Encore: 3D Printed Augmentation of Everyday Objects with Printed-Over, Affixed and Interlocked Attachments. In UIST '15: Proceedings of the 28th annual ACM symposium on User interface software and technology. ACM Press, New York, New York, USA, 73-82.

[18] Xiang 'Anthony' Chen, Jeeeun Kim, Jennifer Mankoff, Tovi Grossman, Stelian Coros, and Scott E Hudson. 2016. Reprise: A Design Tool for Specifying, Generating, and Customizing 3D Printable Adaptations on Everyday Objects. In UIST '16: Proceedings of the 29th annual ACM symposium on User interface software and technology. ACM Press, New York, New York, USA, 29-39.

[19] Nigel Cross. 2004. Expertise in design: an overview. Design Studies 25, 5 (Sept. 2004), 427-441.

[20] Sean Follmer, David Carr, Emily Lovell, and Hiroshi Ishii. 2010. CopyCAD: remixing physical objects with copy and paste from the real world. In UIST '10: Proceedings of the 23nd annual ACM symposium on User interface software and technology. ACM, New York, New York, USA, 381-382.

[21] Sean Follmer and Hiroshi Ishii. 2012. KidCAD: digitally remixing toys through tangible tools. In CHI '12: Proceedings of the SIGCHI Conference on Human Factors in Computing Systems.

[22] Madeline Gannon, Tovi Grossman, and George Fitzmaurice. 2016. ExoSkin: On-Body Fabrication. In CHI '16: Proceedings of the $2016 \mathrm{CHI}$ Conference on Human Factors in Computing Systems. To appear.

[23] Tovi Grossman, George Fitzmaurice, and Ramtin Attar. 2009. A survey of software learnability. In CHI '09: Proceedings of the 27th international conference on Human Factors in Computing Systems. ACM Press, New York, New York, USA, 649-658.

[24] Raymonde Guindon. 1990. Knowledge exploited by experts during software system design. International Journal of Man-Machine Studies 33, 3 (Sept. 1990), 279-304.

[25] Anhong Guo, Jeeeun Kim, Xiang 'Anthony' Chen, Tom Yeh, Scott E Hudson, Jennifer Mankoff, and Jeffrey P. Bigham. 2017. Facade: Autogenerating Tactile Interfaces to Appliances. In CHI '17: Proceedings of the 2017 CHI Conference on Human Factors in Computing Systems.
ACM Press, New York, New York, USA, 5826-5838.

[26] Chun-Heng Ho. 2001. Some phenomena of problem decomposition strategy for design thinking: differences between novices and experts. Design Studies 22, 1 (Jan. 2001), 27-45.

[27] Megan Hofmann, Gabriella Hann, Scott E Hudson, and Jennifer Mankoff. 2018. Greater than the Sum of its PARTs: Expressing and Reusing Design Intent in 3D Models. In CHI '18: Proceedings of the 36th Annual ACM Conference on Human Factors in Computing Systems. ACM, New York, New York, USA, 301-12.

[28] Nathaniel Hudson, Celena Alcock, and Parmit K Chilana. 2016. Understanding Newcomers to 3D Printing: Motivations, Workflows, and Barriers of Casual Makers. In CHI '16: Proceedings of the 2016 CHI Conference on Human Factors in Computing Systems. ACM, New York, New York, USA, 384-396.

[29] Yuki Igarashi and Hiromasa Suzuki. 2011. Cover geometry design using multiple convex hulls. Computer-Aided Design 43, 9 (Sept. 2011), 1154-1162.

[30] Michael D Jones, Kevin Seppi, and Dan R Olsen. 2016. What you Sculpt is What you Get: Modeling Physical Interactive Devices with Clay and 3D Printed Widgets. In CHI '16: Proceedings of the 2016 CHI Conference on Human Factors in Computing Systems. ACM, New York, New York, USA, 876-886.

[31] Jeeeun Kim, Anhong Guo, Tom Yeh, Scott E Hudson, and Jennifer Mankoff. 2017. Understanding Uncertainty in Measurement and Accommodating its Impact in 3D Modeling and Printing. In Proceedings of the 2017 Conference on Designing Interactive Systems. ACM, New York, New York, USA, 1067-1078.

[32] Jarrod Knibbe, Tovi Grossman, and George Fitzmaurice. 2015. Smart Makerspace: An Immersive Instructional Space for Physical Tasks. In Proceedings of the 2015 International Conference on Interactive Tabletops \& Surfaces. ACM, New York, New York, USA, 83-92.

[33] Andrew J Ko, Robin Abraham, Laura Beckwith, Alan Blackwell, Margaret Burnett, Martin Erwig, Chris Scaffidi, Joseph Lawrance, Henry Lieberman, Brad A Myers, Mary Beth Rosson, Gregg Rothermel, Mary Shaw, and Susan Wiedenbeck. 2011. The state of the art in end-user software engineering. ACM Computing Surveys (CSUR) 43, 3 (April 2011), 21-44.

[34] Andrew J Ko, Brad A Myers, and Htet Htet Aung. 2004. Six Learning Barriers in End-User Programming Systems. In 2004 IEEE Symposium on Visual Languages - Human Centric Computing. IEEE, 199-206.

[35] Yuki Koyama, Shinjiro Sueda, Emma Steinhardt, Takeo Igarashi, Ariel Shamir, and Wojciech Matusik. 2015. AutoConnect: computational design of 3D-printable connectors. ACM Transactions on Graphics (TOG) 34, 6 (Nov. 2015), 231-11

[36] Manfred Lau, Masaki Hirose, Akira Ohgawara, Jun Mitani, and Takeo Igarashi. 2012. Situated modeling: a shape-stamping interface with tangible primitives. In TEI '12: Proceedings of the Sixth International Conference on Tangible, Embedded and Embodied Interaction. ACM, New York, New York, USA, 275-282.

[37] David Ledo, Fraser Anderson, Ryan Schmidt, Lora Oehlberg, Saul Greenberg, and Tovi Grossman. 2017. Pineal: Bringing Passive Objects to Life with Embedded Mobile Devices. In CHI '17: Proceedings of the 2017 CHI Conference on Human Factors in Computing Systems. 1-11.

[38] Ghang Lee, Charles M Eastman, Tarang Taunk, and Chun-Heng Ho. 2010. Usability principles and best practices for the user interface design of complex 3D architectural design and engineering tools. International fournal of Human-Computer Studies 68, 1-2 (Jan. 2010), 90-104.

[39] Wei Li, Tovi Grossman, and George Fitzmaurice. 2012. GamiCAD: a gamified tutorial system for first time autocad users. In UIST '12: Proceedings of the 25th annual ACM symposium on User interface software and technology. ACM, New York, New York, USA, 103-112. 
[40] Yangyan Li, Xiaokun Wu, Yiorgos Chrysathou, Daniel Cohen-Or, Yangyan Li, Xiaokun Wu, Yiorgos Chrysathou, Andrei Sharf, and Niloy J Mitra. 2011. GlobFit: consistently fitting primitives by discovering global relations. consistently fitting primitives by discovering global relations, Vol. 30. ACM.

[41] Henry Lieberman, Fabio Paternò, Markus Klann, and Volker Wulf. 2006. End-User Development: An Emerging Paradigm. In End User Development. Springer, Dordrecht, Dordrecht, 1-8.

[42] Thomas Ludwig, Alexander Boden, and Volkmar Pipek. 2017. 3D Printers as Sociable Technologies: Taking Appropriation Infrastructures to the Internet of Things. ACM Transactions on Computer-Human Interaction (TOCHI 24, 2 (April 2017), 17-28.

[43] Samantha McDonald, Niara Comrie, Erin Buehler, Nicholas Carter, Braxton Dubin, Karen Gordes, Sandy McCombe-Waller, and Amy Hurst. 2016. Uncovering Challenges and Opportunities for 3D Printing Assistive Technology with Physical Therapists. In Proceedings of the 18th International ACM SIGACCESS Conference on Computers and Accessibility. ACM, New York, New York, USA, 131-139.

[44] R Martin McGuire and Amy Hurst. 2015. Nickel for Scale. https: //nickelforscale.com/

[45] Brenna McNally, Leyla Norooz, Alazandra Shorter, and Evan Golub. 2017. Toward Understanding Children's Perspectives on Using 3D Printing Technologies in their Everyday Lives. In IDC '17: Proceedings of the 2017 Conference on Interaction Design and Children. ACM, New York, New York, USA, 298-303.

[46] David A Mellis, Leah Buechley, Mitchel Resnick, and Björn Hartmann. 2016. Engaging Amateurs in the Design, Fabrication, and Assembly of Electronic Devices. In Proceedings of the 2016 ACM Conference on Designing Interactive Systems. ACM, New York, New York, USA, 12701281.

[47] Stefanie Mueller, Sangha Im, Serafima Gurevich, Alexander Teibrich, Lisa Pfisterer, François V Guimbretière, and Patrick Baudisch. 2014. WirePrint: 3D printed previews for fast prototyping. In UIST '14: Proceedings of the 27th annual ACM symposium on User interface software and technology. New York, New York, USA, 273-280.

[48] Brad A Myers, Scott E Hudson, and Randy Pausch. 2000. Past, present, and future of user interface software tools. ACM Transactions on Computer-Human Interaction (TOCHI 7, 1 (March 2000).

[49] Donald A Norman. 2013. The Design of Everyday Things. Basic Books.

[50] Lora Oehlberg, Wesley Willett, and Wendy E. Mackay. 2015. Patterns of Physical Design Remixing in Online Maker Communities. In $\mathrm{CHI}$ '15: Proceedings of the 33rd Annual ACM Conference on Human Factors in Computing Systems. New York, New York, USA, 639-648.

[51] John Pane and Brad A Myers. 1996. Usability Issues in the Design of Novice Programming Systems. Technical Report. Carnegie Mellon University.

[52] Jeremiah Parry-Hill, Patrick C Shih, Jennifer Mankoff, and Daniel Ashbrook. 2017. Understanding Volunteer AT Fabricators: Opportunities and Challenges in DIY-AT for Others in e-NABLE. In CHI '17: Proceedings of the 2017 CHI Conference on Human Factors in Computing Systems. ACM, New York, New York, USA, 6184-6194.

[53] Joshua M Pearce. 2015. Applications of Open Source 3-D Printing on Small Farms. Organic Farming 1, 1 (April 2015), 19-35.

[54] Huaishu Peng, Jimmy Briggs, Cheng-Yao Wang, Kevin Guo, Joseph Kider, Stefanie Mueller, Patrick Baudisch, and François V Guimbretière. 2018. RoMA: Interactive Fabrication with Augmented Reality and a Robotic 3D Printer. In CHI '18: Proceedings of the 36th Annual ACM Conference on Human Factors in Computing Systems.

[55] Huaishu Peng, Rundong Wu, Steve Marschner, and François V Guimbretière. 2016. On-The-Fly Print: Incremental Printing While Modelling. In CHI '16: Proceedings of the 2016 CHI Conference on Human Factors in Computing Systems. ACM, New York, New York, USA, 887896.
[56] Romain Prévost, Emily Whiting, Sylvain Lefebvre, and Olga SorkineHornung. 2013. Make it stand: balancing shapes for 3D fabrication. ACM Transactions on Graphics (TOG) 32, 4 (July 2013), 81.

[57] Raf Ramakers, Fraser Anderson, Tovi Grossman, and George Fitzmaurice. 2016. RetroFab: A Design Tool for Retrofitting Physical Interfaces using Actuators, Sensors and 3D Printing. In CHI '16: Proceedings of the 2016 CHI Conference on Human Factors in Computing Systems. ACM, New York, New York, USA, 409-419.

[58] Martin P Robillard and Robert DeLine. 2010. A field study of API learning obstacles. Empirical Software Engineering 16, 6 (Dec. 2010), 703-732.

[59] Greg Saul, Manfred Lau, Jun Mitani, and Takeo Igarashi. 2011. SketchChair: an all-in-one chair design system for end users. In TEI '11: Proceedings of the fifth international conference on Tangible, embedded, and embodied interaction. New York, New York, USA, 73-80.

[60] R Shewbridge, A Hurst, and S K Kane. 2014. Everyday making: identifying future uses for 3D printing in the home. ACM Conference on Designing Interactive Technology (2014), 815-824.

[61] Herbert A Simon. 1973. The structure of ill structured problems. Artificial Intelligence 4, 3-4 (Dec. 1973), 181-201.

[62] Herbert A Simon and simon. 1956. Rational Choice and the Structure of the Environment. Psychological Review 63, 2 (1956), 129-138.

[63] Nagendra G Tanikella, Benjamin Savonen, John Gershenson, and Joshua M Pearce. 2017. Viability of Distributed Manufacturing of Bicycle Components with 3-D Printing: CEN Standardized Polylactic Acid Pedal Testing. Journal of Humanitarian Engineering 5, 1 (May 2017), 3.

[64] Joao Marcelo Teixeira, Gutenberg Barros, Veronica Teichrieb, and Walter Franklin Correia. 2016. 3D Printing as a Means for Augmenting Existing Surfaces. In 2016 XVIII Symposium on Virtual and Augmented Reality (SVR). IEEE, 24-28.

[65] Daniel Tetteroo, Iris Soute, and Panos Markopoulos. 2013. Five key challenges in end-user development for tangible and embodied interaction. In Proceedings of the 15th ACM on International conference on multimodal interaction. ACM, New York, New York, USA, 247-254.

[66] Thingiverse. 2018. https://thingiverse.com. Accessed: 2018-09-18.

[67] TinkerCAD. 2018. https://tinkercad.com. Accessed: 2018-09-18.

[68] David G Ullman, Thomas G Dietterich, and Larry A Stauffer. 1988. A model of the mechanical design process based on empirical data. $A I$ EDAM 2, 1 (Feb. 1988), 33-52.

[69] Nobuyuki Umetani and Ryan Schmidt. 2017. SurfCuit: SurfaceMounted Circuits on 3D Prints. IEEE Computer Graphics and Applications 38, 3 (2017), 52-60.

[70] J Vanek, J A G Galicia, and B Benes. 2014. Clever Support: Efficient Support Structure Generation for Digital Fabrication. Computer Graphics Forum 33, 5 (Aug. 2014), 117-125.

[71] L Wang and E Whiting. 2016. Buoyancy Optimization for Computational Fabrication. Computer Graphics Forum 35, 2 (May 2016), 49-58.

[72] Weiming Wang, Haiyuan Chao, Jing Tong, Zhouwang Yang, Xin Tong, Hang Li, Xiuping Liu, and Ligang Liu. 2015. Saliency-Preserving Slicing Optimization for Effective 3D Printing. Computer Graphics Forum 34, 6 (Sept. 2015), 148-160.

[73] Mian Wei and Karan Singh. 2017. Bend-a-rule: a fabrication-based workflow for 3D planar contour acquisition. In Proceedings of the 1st Annual ACM Symposium on Computational Fabrication. ACM, New York, New York, USA, 5-7.

[74] Christian Weichel, Jason Alexander, Abhijit Karnik, and Hans Gellersen. 2014. SPATA: Spatio-Tangible Tools for Fabrication-Aware Design. In Proceedings of the Ninth International Conference on Tangible, Embedded, and Embodied Interaction. ACM, New York, New York, USA, 189-196. 
[75] Christian Weichel, John Hardy, Jason Alexander, and Hans Gellersen. 2015. ReForm: Integrating Physical and Digital Design through Bidirectional Fabrication. In UIST '15: Proceedings of the 28th annual ACM symposium on User interface software and technology. ACM Press, New York, New York, USA, 93-102.

[76] Christian Weichel, Manfred Lau, David Kim, Nicolas Villar, and Hans W Gellersen. 2014. MixFab: A Mixed-Reality Environment for Personal Fabrication. In CHI'14: Proceedings of the SIGCHI Conference on Human Factors in Computing Systems. ACM Press, New York, New York, USA, 3855-3864.

[77] Jun Wu, Christian Dick, and Rudiger Westermann. 2015. A System for High-Resolution Topology Optimization. IEEE Transactions on Visualization and Computer Graphics 22, 3 (2015), 1195-1208.
[78] Yue Xie, Weiwei Xu, Yin Yang, Xiaohu Guo, and Kun Zhou. 2015. Agile structural analysis for fabrication-aware shape editing. Computer Aided Geometric Design 35-36 (May 2015), 163-179.

[79] Amanda K. Yung, Zhiyuan Li, and Daniel Ashbrook. 2018. Printy3D: In-situ Tangible Three-dimensional Design for Augmented Fabrication. In Proceedings of the 17th ACM Conference on Interaction Design and Children (IDC '18). ACM, New York, NY, USA, 181-194. https://doi. org/10.1145/3202185.3202751

[80] Kening Zhu, Alexandru Dancu, and Shengdong Shen Zhao. 2016. FusePrint: A DIY 2.5D Printing Technique Embracing Everyday Artifacts. In Proceedings of the 2016 ACM Conference on Designing Interactive Systems. ACM, New York, New York, USA, 146-157. 